In addition to confirming the hypothetical helical configuration of human chromosomes, this study demonstrated that secondary constrictions are less tightly coiled regions of the chromonemata. Moreover, this technique offers the possibility of a more quantitative analysis of chromosomos at the chromonema level. If a reproducible gyre number is established, coil counts may bocome a more securate method of determining arm ratios. The analysis of chromosome breaks or translocations, induced by treatment with chemicals or ionizing radiation, may be described more accurately with partial dissociation of the chromosomes. Quantitative studies regarding the number of helices and the measurement of the length and diameter of the coils are now in progress and will be published elsewhore.

This work was aided by grant $G B-15$ from the U.S. National Science Foundation, administered by Dr. Donald E. Rounds. I thank him for his advice.

This communication is dedicated to Prof. Sajiro Makino, Zoological Institute, Hokkaido University, Sapporo, Japan, in honour of his sixtieth birthday on June 21, 1966.

Pasadona Foundation for Medical Research, YASUSHI OHNUKI Pasadena, California.

${ }^{1}$ Swanson, C. P., Cytology and Cytogenetics (Prentice-Hall, Inc., Englewood Cliffs, N.J., 1957).

- Bonner, J., and Ts'o, P. (eds.), The Nucleohistones (Holden-Day, Inc, San Francisco, 1964).

${ }^{3}$ Saksela, E., and Moorhead, P. S., Cytogenetics, 1, 225 (1962).

1 Ferguson-Smith, M. A., Ferguson-Smith, M. E., Ellis, P. M., and Dickson, M., Cytogenetics, 1, 325 (1962).

'Sasaki, M. S., and Makino, S., Amer. J. Human Genet., 15, 24 (1963).

- Matsuura, H., Tanifuji, S., Saho, T., Iwabuchi, M., and Takizawa, S., J. Fac. Scs. Hokkaido Univ., Ser. V, 8, 201 (1962).

\title{
GENETICS
}

\section{Chimerical Nature of the Entire-leaf Variant in the Potato Variety 'Majestic'}

THe docken, or entire-leaf variation (Fig. 1), like several other variations for leaf shape in potatoes, has not been shown previously to be a chimera. Leaf shape in potatoes is largely determined by the constitution of $L_{2}$ (ref. 1), and the two standard methods of investigating potato chimeras, breeding behaviour and eye-excision, give information on $L_{\mathrm{a}}$ in the variant and not on $L_{1}$. It is thus not possible by these two methods to show that $L_{1}$ in a variant is unchanged. Information of $L_{1}$ may, however, be obtained by X-ray treatment ${ }^{2}$.

Crossing 'Majestic' docken-leaf with 'Ulster Prince' resulted in a family with a segregation of 23 seedlings with entire leaves: 17 seedlings with compound, pinnate leaves

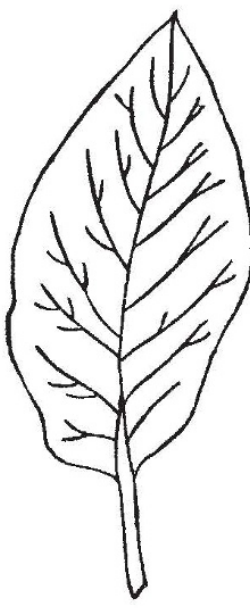

$a$

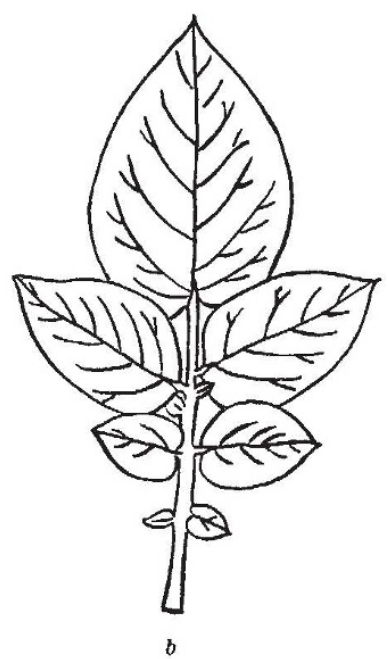

Fig. 1. (a) Docken-leaf and (b) normal, compound pinnate leaf of shoots (the control cross of normal 'Majestic' with 'Ulster Prince' gave 53 seedlings with compound, pinnate leaves and none with entire leaves).

Eye-excision experiments with docken-loaf tubers produced only plants with entire leaves, no normal 'Majestic' plants being obtained. Root buds, produced by the method of Howard ${ }^{3}$, also resulted only in plants with entire leaves. It thus seems that docken-leaf in 'Majestic' results from a mutation for entire leaves in tho $L_{2}$ layer and that the $L_{3}$ layer also now has the same constitution as that of $L_{2}$; a similar replacement of $L_{3}$ by $L_{3}$ cells has been observed in triploid-hexaploid potato chimeras ${ }^{1}$.

Five apical (rose) ends of tubers of docken-leaf 'Majestic' were given an X-ray dosage of 3,500 r. Four of them produced plants and one of the four had a mixture of normal and docken-leaf shoots. The production of the shoots with normal leaves was presumably because in the docken-leaf variant $L_{1}$ is still normal, unchanged 'Majestic'. The frequency (about 12.5 per cent) of shoots with all layers with the constitution of $L_{1}$ in the variant is similar to that of full 'Red King' from normal 'Red King' and of white from 'Bonte' sports after X-ray treatmont ${ }^{2}$.

The finding that the docken-leaf sport is a chimera in which $L_{1}$ is still normal 'Majestic' suggests that certain other variants, such as wildings, feathery wildings and bolters, which do not appear to be chimeras from the results of eye-exeision experiments, may be chimeras in which $L_{2}$ and $L_{3}$ have the mutated character but in which $L_{1}$ is still unchanged.

H. W. HOW ARD

Plant Breeding Institute,

Trumpington, Cambridge.

${ }^{2}$ Howard, H. W., Wainwright, J., and Fuller, J. M., Genetica, 34, 118 (1963)

${ }^{2}$ Howard, H. W., Rad. Bot., 4, 361 (1964).

${ }^{8}$ Howard, H. W., Nature, 203, 1303 (1964).

\section{Estimation of the Frequency of Functioning Gametes in Monosomics}

We regret that the estimates of $p$ and $q$ of the function ing male gametes with $n$ and $(n-1)$ chromosomes respect ively given in our recent communication ${ }^{1}$ are not consis tent. The correct estimates should be as follows.

On applying the maximum likelihood method, the estimate of $p$ may be obtained by taking the positive root of the quadratic equation:

$p^{2}\left[N\left(p^{\prime}-q^{\prime}\right)\right]+p\left[q^{\prime}\left(a_{1}+a_{2}\right)-p^{\prime}\left(N+a_{1}\right)\right]+a_{1} p^{\prime}=0$

and the variance of the estimate will be:

$$
V_{p}=\frac{p^{2} q^{2}\left(p^{\prime} q+p q^{\prime}\right)^{2}}{\left(p^{\prime} q+p q^{\prime}\right)^{2}\left(a_{1} q^{2}+a_{3} p^{2}\right)+a_{2} p^{2} q^{2}\left(q^{\prime}-p^{\prime}\right)^{2}}
$$

where $a_{1}, a_{2}$ and $a_{3}\left(a_{1}+a_{2}+a_{3}=N\right)$ are the observed frequencies of disomic, monosomic and nullisomic respectively and $p^{\prime}$ and $q^{\prime}\left(p^{\prime}+q^{\prime}=1\right)$ are the relative frequencies of the functioning female gametes with $n$ and $(n-1)$ chromosomes respectively.

The square root of $V_{p}$ will give the standard orror (S.E.) of the estimate.

It can be shown that:

$$
\begin{aligned}
& \text { when } p^{\prime}=q^{\prime}=\frac{1}{2} \\
& \qquad p=\frac{2 a_{1}}{N} \text { and } q=\frac{2 a_{3}}{N}
\end{aligned}
$$

and the variance of the estimate will be reduced to $\frac{2 p q}{N}$.

By using (1) in our numerical example ${ }^{1}$, we obtain:

$$
-59 \cdot 28 p^{2}+12 \cdot 08 p+3 \cdot 12=0
$$

giving $p=0.3529$ and $q=1-p=0.647 \mathrm{I}$.

Similarly, from (2), we have

$$
V_{p}=0.00324967
$$

and $S . E .=0.0570$

It may be observed that the estimate of $p$ is not significantly differ ent from the experimental results ${ }^{2} 0 \cdot 39$. 